\title{
Comparing the effectiveness of revascularization interventions with medical therapy in patients with ischemic cardiomyopathy: A systematic review and meta-analysis
}

\author{
Saeed Bagheri Faradonbeh ${ }^{1}$, Farbod Ebadi Fard Azar*2, Aziz Rezapour ${ }^{2}$, Marjan Hajahmadi², Seyed Javad Hajmiresmail ${ }^{3}$
}

Received: 10 Apr 2018

Published: 19 Dec 2018

\section{Abstract}

Background: Determining the effectiveness of cardiovascular interventions plays an important role in reimbursement decisions, health care pricing, and providing clinical guidance on the use of existing clinical technologies. This study aimed to review and analyze the effectiveness of revascularization interventions (CABG and PCI) compared to medical therapy in patients with ischemic cardiomyopathy.

Methods: Different databases were searched up to December 2017. The articles were selected based on inclusion and exclusion criteria. Quality of all studies was evaluated by Jadad score and relevant checklists. The I2 test was used to test heterogeneity. Also, to integrate the results of similar studies, meta-analysis was done using STATA software.

Results: A total of 18 studies were included. Based on the random effects model, the overall results of comparing the effectiveness of revascularization interventions with medical therapy were as follow: 38.94 [95\% CI: 26.95-50.94, $\mathrm{p}<0.001, \mathrm{I}^{2}=99.6 \%, \mathrm{p}<0.001$ ], [75.31, 95\% CI: 74.06-76.57, $\mathrm{p}<0.001, \mathrm{I}^{2}=88.8, \mathrm{p}<0.001$ ], and 75.76 [95\% CI: 71.99-79.53, $\left.\mathrm{p}<0.001, \mathrm{I}^{2}=99.2, \mathrm{p}<0.001\right]$ for cardiac mortality rate, quality of life, and 5-year survival, respectively. Also, in patient satisfaction index, revascularization interventions were shown to be more effective than medical therapy.

Conclusion: This study showed that revascularization interventions in all studied indices were more effective than medical therapy. Also, between revascularization interventions, PCI was more effective in cardiovascular mortality and 5-year survival than CABG in terms of quality of life. Moreover, CABG was more effective than PCI. In patient satisfaction index, the results of the 2 included studies were contradictory.

Keywords: Effectiveness, Revascularization, Medical therapy, Ischemic cardiomyopathy

Conflicts of Interest: None declared

Funding: Iran University of Medical Sciences, Tehran, Iran

\section{*This work has been published under CC BY-NC-SA 1.0 license.}

Copyright $\odot$ Iran University of Medical Sciences

Cite this article as: Bagheri Faradonbeh S, Ebadi Fard Azar F, Rezapour A, Hajahmadi M, Hajmiresmail SJ. Comparing the effectiveness of revascularization interventions with medical therapy in patients with ischemic cardiomyopathy: A systematic review and meta-analysis. Med $J$ Islam Repub Iran. 2018 (19 Dec);32:127. https://doi.org/10.14196/mjiri.32.127

\section{Introduction}

Ischemic cardiomyopathy is generally defined as left ventricular dysfunction due to severe coronary artery disease (CAD), myocardial infarction, or ongoing ischemic injury (hibernating myocardium) (4).

Despite major advances in cardiovascular therapy, cardiovascular disease remains the primary cause of death

Corresponding author: Dr Farbod Ebadi Fard Azar, ebadifardazar.f@iums.ac.ir

1. Department of Health Economics, School of Health Management and Information Sciences, Iran University of Medical Sciences, Tehran, Iran

2. Health Management and Economics Research Center, Iran University of Medical Sciences, Tehran, Iran

3. Department of Cardiology, Iran University of Medical Sciences, Tehran, Iran and disability in the industrialized world. Unfortunately, improved survival after acute myocardial infarction (MI) has been associated with an increased incidence of ischemic cardiomyopathy and heart failure. Patients with ischemic cardiomyopathy, defined as impaired left ventricular (LV), have high mortality rates and an impaired

$\uparrow$ What is "already known" in this topic:

To date, no systematic review study has been done to compare the effectiveness of revascularization interventions (CABG and $\mathrm{PCI}$ and medical therapy in patients with ischemic cardiomyopathy.

$\rightarrow$ What this article adds:

The results of this study showed that revascularization interventions in all studied indices were more effective than medical therapy. 
quality of life (6).

There are several methods for treating and controlling ischemic cardiomyopathy in different health systems in the world: (A) medical therapy (MT) which uses anticoagulants, such as thrombolytic, beta blocker, calcium blocker, antiarrhythmic, nitrates, diuretics, antiplatelet, and lipid regulating drugs; (B) percutaneous coronary intervention (PCI) that includes piercing the skin to access the femoral artery by a catheter, catheter-guided balloon to narrow or block the coronary artery, inflating the balloon, and dilating coronary stenting to prevent reblockage; and (C) coronary artery bypass graft (CABG) that includes a bypass with saphenous vein or arterial graft pieces of breast through open narrowed or blocked coronary sternum in place. This is a surgical technique that involves opening the chest and the tight and closed coronary artery and is usually done using a vein or artery from other parts of the body (9-10).

The advantages of the surgical technique include angina relief in $60 \%$ to $90 \%$ of patients in the first year, a significant reduction in mortality due to cardiovascular disease when combined with drug therapy, and a reduction in revascularization after 1 year. However, its disadvantages are high costs- in particular hospital care costs- and an increase in myocardial infarction rate compared to drug therapy (13).

To date, no systematic reviews have been made on this topic. Medical treatment for coronary disease has advanced dramatically in recent years and produced prognostic benefits in the context of properly designed, randomized, controlled trials. Surgical techniques have also advanced, however, it is difficult to ensure that they have truly reduced mortality rate, as such comparisons are retrospective rather than concurrent. Even if the proportionate benefits from surgery were to increase, the falling mortality with optimal medical therapy would reduce the absolute benefits of surgery over medical treatment. Thus, surgical interventions seem to become outdated, and thus their relevance to modern medical practice must be questioned (16-17). A clinical trial study compared drug therapy with surgery in patients with ischemic heart disease and found no significant evidence on the benefit of these 2 therapies in reducing mortality and morbidity (10).

Performing systematic review studies and determining the effectiveness of cardiovascular interventions play an important role in reimbursement decisions, health care pricing, providing clinical guidance on the use of existing clinical technologies, strategic purchasing of interventions, providing targeted health care, and producing scientific evidence for policy-making and, ultimately, the optimal allocation of financial resources of the health sector to cardiovascular diseases $(10,20)$. Thus, the aim of this study was to review and compare the effectiveness of revascularization interventions (CABG and $\mathrm{PCI}$ ) and medical therapy in patients with ischemic cardiomyopathy.

\section{Review of the literature}

Protocol and registration

This systematic review protocol was registered in PROSPERO International Prospective Register of Sys- tematic Reviews (registration number: CRD42018079889) and published in Medicine (2018) 97:10(e9958).

\section{Study characteristics}

Data were extracted and presented according to PRISMA (providing innovative service models and assessment) criteria (22).

This systematic review included observational (case report, case series, cross sectional, case-control, cohort, etc.) and interventional (quasi-experimental studies, randomized controlled trials, etc.) studies in English and Persian language and examined the effectiveness of revascularization and medical therapy interventions in patients with ischemic cardiomyopathy. Animal studies were not considered.

\section{Participants}

This systematic review targeted studies conducted on ischemic cardiomyopathy patients with ejection fraction $<$ $35 \%$ who underwent percutaneous coronary intervention, surgery, or medical treatment.

\section{Report characteristics}

Only studies that had abstracts in English and those studies whose full-texts were available were selected. No limitation was considered for date of acceptance or publication. Also, only articles that were published or in press were considered.

\section{Information sources}

Our sources of information included electronic databases, trial registries, and different types of grey literature. An electronic search was performed in PubMed, Cochrane library, Scopus, Web of Science, EMBASE, Tufts Medical Center Cost-Effectiveness Analysis Registry, and NHS Economic Evaluations Database up to 12/30/2017. To identify appropriate key words, in addition to MESH terms, popular and commonly-used phrases stated in the related literature were used. For example, the search strategy in PubMed database was as follows:

1) Percutaneous coronary intervention [MeSH Terms]

2) Coronary artery bypass [MeSH Terms]

3) $\mathrm{CABG}$ [Title/Abstract]

4) Drug therapy [MeSH Terms]

5) Medical therapy [Title/Abstract]

6) Medical treatment [Title/Abstract]

7) Cardiomyopathies [MeSH Terms]

8) Cardiomyopathy [Title/Abstract]

9) Ischemia [Title/Abstract]

10) 1 OR 2 OR 3

11) 4 OR 5 OR 6

12) 7 OR 8 OR 9

13) 10 AND 11 AND 12

First, the search strategy was developed and completed in PubMed, then; a modified strategy was applied to other databases. Other sources were searched to identify related grey literature. ProQuest was searched for dissertations. Meeting abstracts were searched through SCOPUS and Web of Science. Also, reference lists of included studies 
were searched.

\section{Selection process}

Two authors independently performed the primary article screening. First, they reviewed the titles and abstracts of the articles independently. Then, the selected articles were categorized into 2 groups: relevant and irrelevant. Articles categorized as irrelevant by both reviewers were eliminated from the study. Then, each reviewer reviewed the full-text of the remaining articles and made a list. Then, the 2 lists were compared and nonconformities were discussed. In case of a disagreement, the entire team made the final decision.

\section{Data management}

Data were extracted from papers and entered into data sheets independently by 2 reviewers. These 2 sheets and their differences were checked by a third reviewer. Any potential difference of opinion among reviewers was discussed by the team members.

\section{Data extraction}

The following information was extracted from each article: article ID; author; publication year; study design; sample size; and our final outcomes, including heart mortality rate, survival rate, quality of life, and patient satisfaction (utility) rate. Heart mortality rate was the number of deaths during a particular period of time among patients with ischemic cardiomyopathy (26). The 5-year survival rate is the percentage of people who lived at least 5 years after being diagnosed with a certain disease $(26,28)$. Quality of life is the degree to which an individual is healthy, comfortable, and able to participate in or enjoy life events (29-31). Patient satisfaction is an important and commonly used indicator for measuring the quality of health care, which affects clinical outcomes, patient retention, and medical malpractice claims (33).

\section{Quality assessment}

Studies on quality of life were evaluated by the Jadad score, with a score between 0 and 5 based on randomization, blindness, a decrease in the number of samples during the study, and relevant checklists (34-35). To test heterogeneity, the $\mathrm{I}^{2}$ test was used, and as there was heterogeneity or lack of studies, the random effects method was used. Percentages of around 25\% $\left(\mathrm{I}^{2}=25\right), 50 \%\left(\mathrm{I}^{2}=50\right)$, and $75 \%\left(\mathrm{I}^{2}=75\right)$ were recognized as low, medium, and high heterogeneity, respectively (36). The funnel plot was used to detect publication bias in studies included in the meta-analysis, but there was no publication bias, as the distribution of studies was symmetric.

\section{Data synthesis}

To integrate the results of studies with similar results, meta-analysis was done using STATA software. Also, the results of the studies were analyzed based on the ES relevant outcome. Significance level was set at $p<0.05$ (37).

\section{Results}

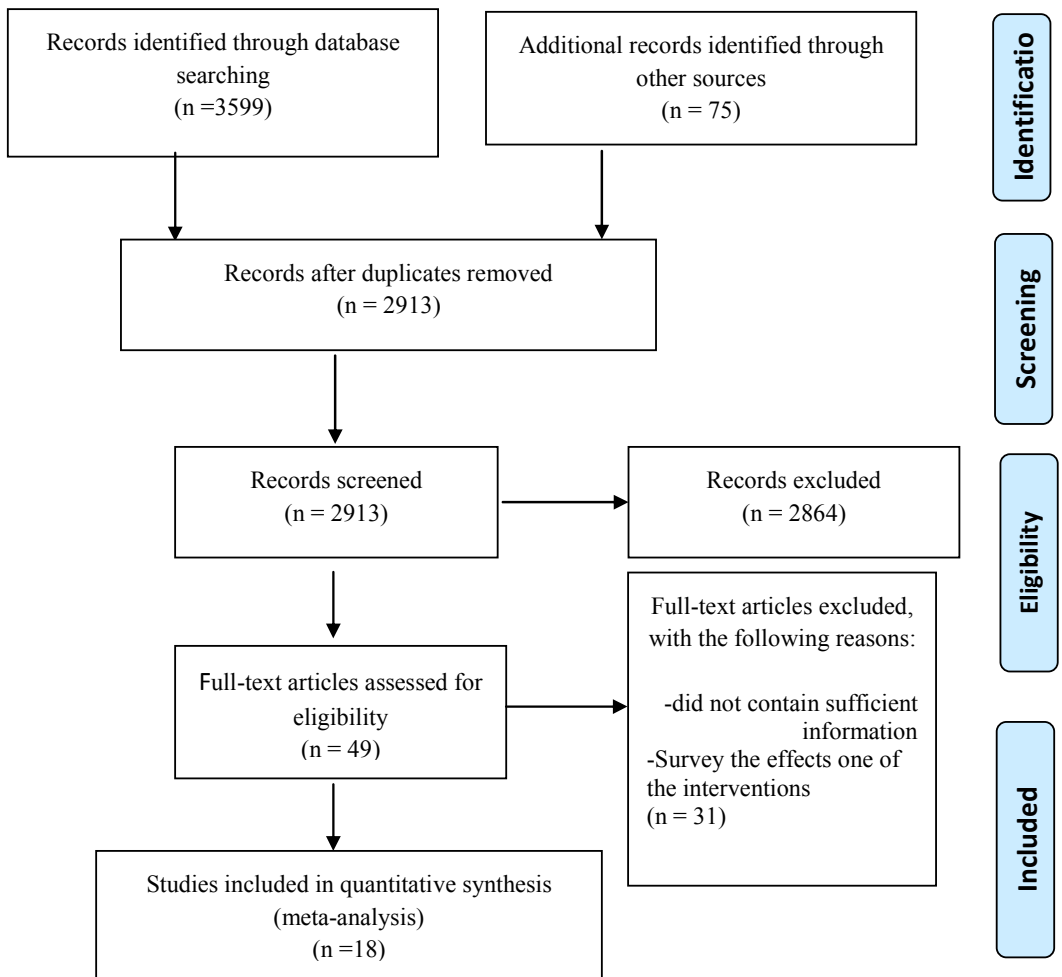

Fig. 1. Flow diagram showing the process of study selection 
Comparing the effectiveness of revascularization interventions with medical therapy

Table 1. The main characteristics studies included

\begin{tabular}{|c|c|c|c|c|c|c|c|c|c|}
\hline Effectiveness & Study & Year & Study design & Country/city & $\begin{array}{c}\mathrm{MT} \\
\% \\
\end{array}$ & $\begin{array}{c}\text { CABG } \\
\% \\
\end{array}$ & $\begin{array}{c}\text { PCI } \\
\% \\
\end{array}$ & $\begin{array}{c}\text { Sample } \\
\text { size }\end{array}$ & \\
\hline \multirow{9}{*}{ Heart mortality } & Harindra C (1) & 2011 & Cohort & Canada & 75 & 70 & 73 & 39131 & \\
\hline & Akira Marui (2) & 2016 & $\mathrm{RCT}$ & Japan & 49.3 & 40.5 & & 1212 & \\
\hline & Francesco Nudi (3) & 2017 & Observational & Italy & 80 & 20 & 20 & 3786 & $\mathrm{df}=17$ \\
\hline & Eric J. Velazquez (5) & 2011 & Cohort & North Carolina & 43 & 29 & & 368 & $I^{2}=99.6 \%$ \\
\hline & Terence Lin (7) & 2010 & $\mathrm{RCT}$ & Loma Linda & 3 & 6 & & 1369 & $\mathrm{p}<0.001$ \\
\hline & William E. Boden (8) & 2007 & RCT & New York & 8.3 & & 7.6 & 2287 & $\mathrm{z}=6.36$ \\
\hline & Peter Carson (11) & 2013 & $\mathrm{RCT}$ & USA & 31.4 & 26.6 & & 1212 & \\
\hline & Sahil Khera (12) & 2017 & RCT & New York & 58 & 47 & & 1212 & \\
\hline & Michelle M (14) & 2002 & RCT & Canada & 12.5 & 9.8 & 9.8 & 983 & \\
\hline Total & & & & & 49.87 & 34.46 & 26.87 & 51560 & \\
\hline \multirow{9}{*}{ Survival } & Harindra $\mathrm{C}$ & 2011 & Cohort & Canada & 88 & 92 & 92 & 39131 & \\
\hline & Christopher M (15) & 2002 & Observational & North Carolina & 37 & 61 & & 1841 & \\
\hline & Jacques Claude (18) & 2004 & RCT & Switzerland & 38 & 79 & & 188 & $\mathrm{df}=21$ \\
\hline & Sandra Weiss (19) & 2015 & RCT & USA & 87 & & 88.3 & 2280 & $\mathrm{I}^{2}=99.2 \%$ \\
\hline & Vijay Raja (21) & 2014 & $\mathrm{RCT}$ & Texas & 61 & & 69 & 213 & $\mathrm{p}<0.001$ \\
\hline & William E. Boden (8) & 2007 & RCT & New York & 87 & & 90 & 2287 & $\mathrm{z}=39.38$ \\
\hline & Bimal R. Shah (23) & 2002 & $\mathrm{RCT}$ & Minneapoli, Minn & 47 & 73 & 73 & 580 & \\
\hline & Deborah H (24) & 2012 & RCT & USA & 53 & 75 & 75 & 695 & \\
\hline & Michelle M (14) & 2002 & $\mathrm{RCT}$ & Canada & 91 & 95 & 93 & 983 & \\
\hline Total & & & & & 65.92 & 79.35 & 85.17 & 48198 & \\
\hline \multirow{3}{*}{ QoL } & Daniel B. Mark (25) & 2014 & RCT & North Carolina & 74 & 77 & & 1212 & $\mathrm{df}=7$ \\
\hline & Michelle M. Graham (27) & 2006 & $\mathrm{RCT}$ & Canada & 75.1 & 77.4 & 76 & 2940 & $\mathrm{I}^{2}=88.8 \%$ \\
\hline & Sara Michelly (32) & 2017 & $\mathrm{RCT}$ & Brazil & 70.8 & 75.2 & 76 & 579 & $\begin{array}{c}\mathrm{p}<0.001 \\
\mathrm{z}=117.37\end{array}$ \\
\hline Total & & & & & 73.18 & 76.70 & 76 & 4731 & \\
\hline \multirow{2}{*}{$\begin{array}{l}\text { Patient satisfaction } \\
\text { and utility }\end{array}$} & Michelle M. Graham (27) & 2006 & $\overline{\mathrm{RCT}}$ & Canada & 86.6 & 88.6 & 87.4 & 2940 & Descriptive \\
\hline & Sara Michelly (32) & 2017 & RCT & Brazil & 75.5 & 78 & 80.9 & 579 & \\
\hline
\end{tabular}

In this review, 2913 records were retrieved from electronic databases and manual searches. Search and study selection procedures are summarized in the PRISMA flow-diagram (Fig. 1). After removing duplicate articles, 2864 full-texts were assessed for eligibility. A total of 49 records were reviewed for full-text articles, of which 18 were included in the final analysis. Of the included studies, 23 compared PCI with CABG intervention or effects of one of them, and 8 studies did not contain sufficient information to extract related data.

All the included studies were published between 1970 and 2017. A total of 108008 patients were included in the meta-analysis. All the patients included in the metaanalysis had ischemic cardiomyopathy. PCI arm included 39 012, CABG arm 24 439, and medical therapy arm 44 557 ischemic cardiomyopathy patients. The mean age of the participants was 66.09 years, and average proportions of patient characteristics showed that $77.1 \%$ of the participants were male. All 18 studies were coded as having low risk of bias within all categories.

Because of the great heterogeneity of the studies, the random-effects model and sensitivity analyses approach were selected and 3 studies (Terence Lin (7), William E. Boden (8) and Michelle M (14)) were excluded. Also, the impact of removing each of the studies was evaluated in the summary results but the results did not change. In this meta-analysis, both $\chi^{2}$ based Q-statistic test and $\mathrm{I}^{2}$ test were considered to assess heterogeneity across studies, and $\mathrm{P}$-value less than or equal to 0.05 was considered significant. $\mathrm{I}^{2}$ is a description of the variation across studies that is due to heterogeneity instead of chance.

Mortality rate in ischemic cardiomyopathy patients who underwent $\mathrm{CABG}$ was 34.46 [95\% CI: 23.45-45.82, $\left.\mathrm{p}<0.001, \mathrm{I}^{2}=98.1 \%, \mathrm{p}<0.001\right]$. This index in the PCI intervention was [26.79]95\% CI: 12.92-40.66, $\mathrm{p}<0.001, \mathrm{I}^{2}=$ $99.1 \%, \mathrm{p}<0.001]$, and it was $49.87[95 \% \mathrm{CI}: 27.78-71.97$, $\left.\mathrm{p}<0.001, \mathrm{I}^{2}=99.6 \%, \mathrm{p}<0.001\right]$ for MT. The overall result for this outcome was 38.94 [95\% CI: 26.95-50.94, $\left.\mathrm{p}<0.001, \mathrm{I}^{2}=99.6 \%, \mathrm{p}<0.001\right]$. (Table 1, Fig. 2).

In the context of comparing revascularization interventions with medical therapy in quality of life, we concluded that this index was 76.71 [95\% CI: 75.22-78.72, $\mathrm{p}<0.001$, $\left.\mathrm{I}^{2}=56 \%, \mathrm{p}=0.1\right]$ for CABG. Also, in the PCI intervention, this index was equal to 76 [95\% CI: 75.51-76.49, $\mathrm{p}=0.00$, $\left.\mathrm{I}^{2}=0, \mathrm{p}=1\right]$ and it was $73.18[95 \% \mathrm{CI}$ : 70.24-76.12, $\left.\mathrm{p}<0.001, \mathrm{I}^{2}=74.4, \mathrm{p}=0.02\right]$ for $\mathrm{MT}$. The overall result for Qol (Quality of life) was 75.31 [95\% CI: 74.06-76.57, $\left.\mathrm{p}<0.001, \mathrm{I}^{2}=88.8, \mathrm{p}<0.001\right]$ (Table 1, Fig. 3).

In patient satisfaction and utility of therapeutic interventions, after searching for studies, we eventually came to 2 articles: Michelle M. Graham et al reported that patient satisfaction was $86.6 \%, 88.6 \%$, and $87.4 \%$ for MT, CABG, and PCI, respectively. Moreover, Sara Michelly et al concluded that this index was $75.5 \%, 78 \%$, and $80.9 \%$ for MT, CABG, and PCI, respectively.

According to Table 1 and Figure 4, the 5-year survival rate for PCI was 85.18 [95\% CI: 81.63-88.72, $\mathrm{p}<0.001$, $\left.\mathrm{I}^{2}=94 \%, \mathrm{p}<0.001\right]$. Also, this rate was $79.35[95 \% \mathrm{CI}$ : 68.76-89.95, $\left.\mathrm{p}<0.001, \mathrm{I}^{2}=97.5, \mathrm{p}<0.001\right]$ for $\mathrm{CABG}$ and it was 65.93 [95\% CI: 54.27-77.59, $\mathrm{p}<0.001, \mathrm{I}^{2}=99.6 \%$, $\mathrm{p}<0.001]$ for MT. Also, the overall result for 5-year survival was 75.76 [95\% CI: 71.99-79.53, $\mathrm{p}<0.001, \mathrm{I}^{2}=99.2$, $\mathrm{p}<0.001]$. 


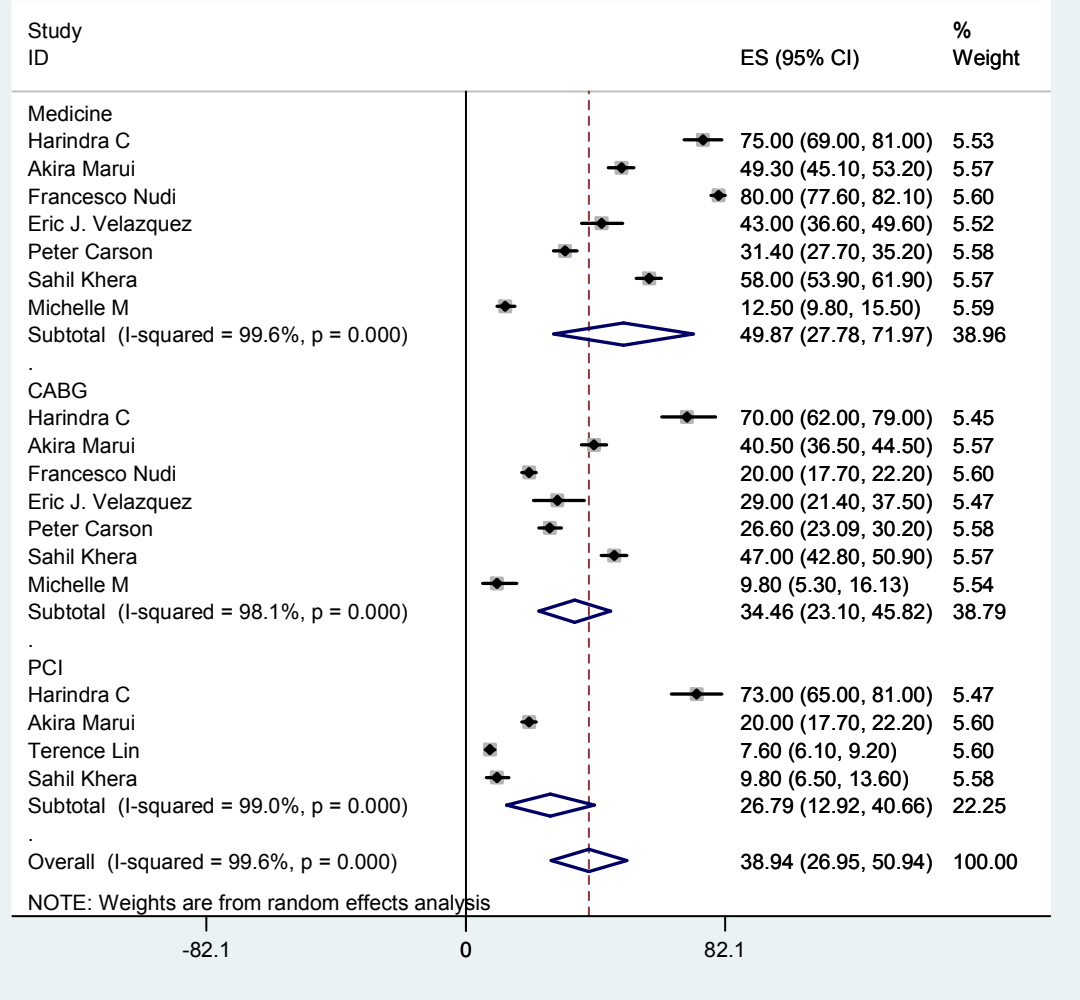

Fig. 2. The Forest plot of ischemic cardiomyopathy mortality in PCI, CABG, and medical therapy interventions

\begin{tabular}{|c|c|c|c|}
\hline \multicolumn{3}{|l|}{ Study } & \multirow{2}{*}{$\begin{array}{l}\% \\
\text { Weight }\end{array}$} \\
\hline ID & & ES $(95 \% \mathrm{Cl})$ & \\
\hline \multicolumn{4}{|l|}{ Medicine } \\
\hline Daniel B. Mark (2014) & $\rightarrow$ & $74.00(70.20,77.30)$ & 7.61 \\
\hline Michelle M. Graham (2006) & + & $75.10(72.60,77.40)$ & 11.35 \\
\hline Sara Michelly (2017) & $\rightarrow 1$ & $70.80(68.00,72.00)$ & 12.99 \\
\hline Subtotal (1-squared $=74.4 \%, \mathrm{p}=0.020)$ & $\diamond$ & $73.18(70.24,76.12)$ & 31.95 \\
\hline \multirow{2}{*}{\multicolumn{4}{|c|}{ CABG }} \\
\hline & & & \\
\hline Daniel B. Mark (2014) & + & $77.00(73.50,80.30)$ & 8.01 \\
\hline Michelle M. Graham (2006) & - & $77.40(77.00,77.70)$ & 19.06 \\
\hline Sara Michelly (2017) & $\rightarrow$ & $75.20(72.00,76.00)$ & 12.99 \\
\hline Subtotal (1-squared $=56.0 \%, \mathrm{p}=0.103)$ & $\theta$ & $76.71(75.20,78.22)$ & 40.07 \\
\hline \multirow{2}{*}{\multicolumn{4}{|c|}{$\mathrm{PCl}$}} \\
\hline & & & \\
\hline Michelle M. Graham (2006) & - & $76.00(75.60,76.60)$ & 18.77 \\
\hline Sara Michelly (2017) & $\rightarrow$ & $76.00(72.00,78.00)$ & 9.21 \\
\hline Subtotal (1-squared $=0.0 \%, p=1.000)$ & 1 & $76.00(75.51,76.49)$ & 27.98 \\
\hline & & & \\
\hline Overall (1-squared $=88.8 \%, \mathrm{p}=0.000$ ) & 0 & $75.31(74.06,76.57)$ & 100.00 \\
\hline \multicolumn{4}{|l|}{ NOTE: Weights are from random effects analysis } \\
\hline $\begin{array}{c}1 \\
-80.3\end{array}$ & 80 & & \\
\hline
\end{tabular}

Fig. 3. The Forest plot of quality of life in PCI, CABG, and medical therapy in ischemic cardiomyopathy

\section{Discussion}

To our knowledge, this study was the first comprehensive systematic review and meta- analysis to compare the effectiveness of revascularization interventions and medical therapy in patients with ischemic cardiomyopathy.

Ultimately, 18 studies were included and effectiveness indicators, such as cardiac death rate, 5 -year survival, and quality of life, were analyzed and patient satisfaction results were reported. In studies included in quantitative synthesis (meta-analysis), 2 examined effectiveness indicators, including cardiac mortality and survival $(1,5)$, and 2 studies done on quality of life examined the utility and 


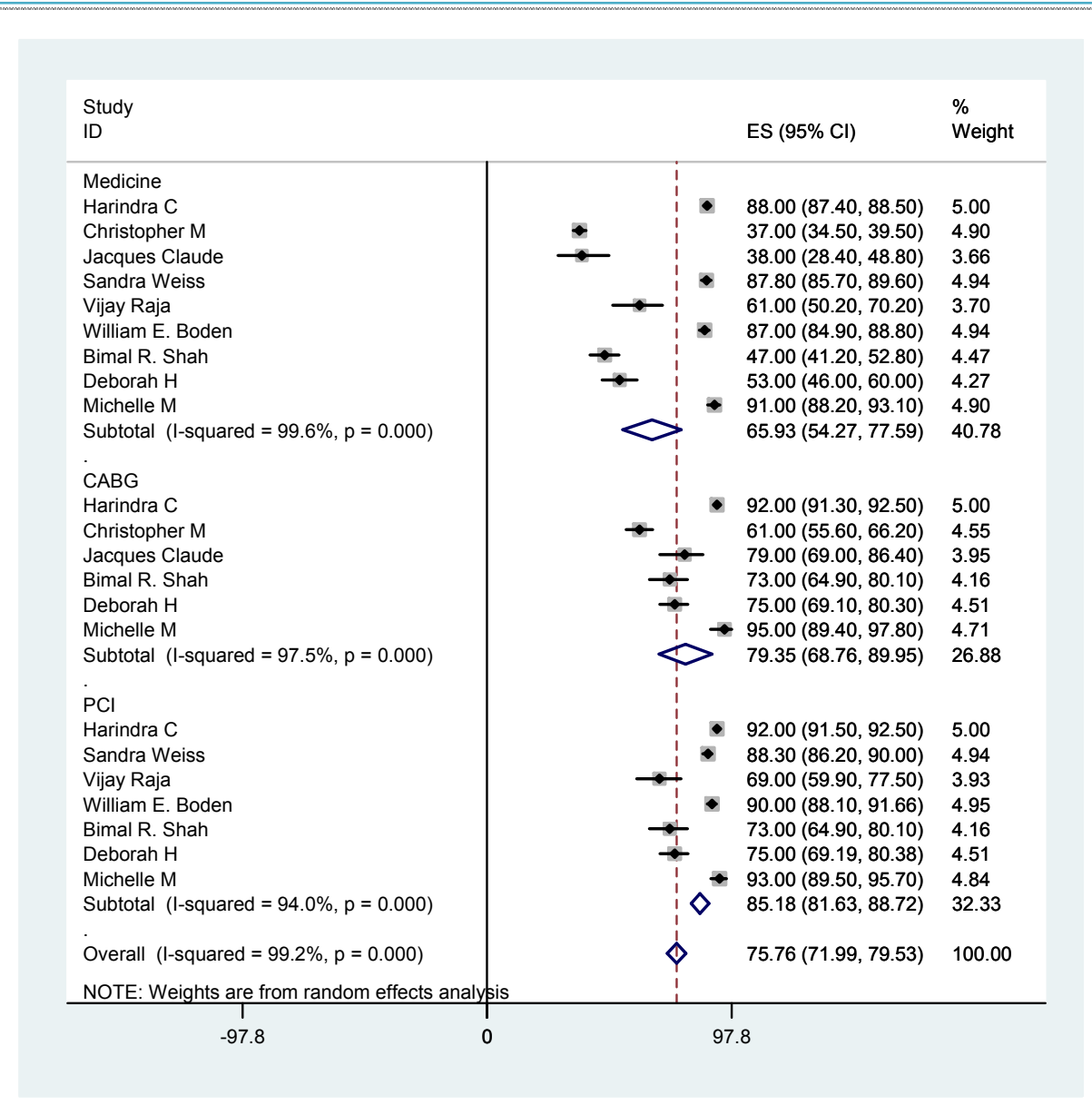

Fig. 4. The Forest plot of 5-year survival in PCI, CABG, and medical therapy in ischemic cardiomyopathy

satisfaction of the patients $(27,32)$.

Revascularization interventions in ischemic cardiomyopathy patients are more effective than medical therapy in preventing cardiac mortality. Also, cardiac mortality rate in PCI was lower than CABG intervention and, as a result, PCI was more effective than CABG. Pursnani et al, in a systematic review and meta-analysis of 12 randomized trials, compared PCI and MT in 7182 stable ischemic heart disease patients and found a strong trend for lower mortality with PCI (38). Schömig et al reported similar trends from 17 trials of PCI versus OMT in 7513 stable patients with ischemia (some with recent MI). They found that PCI was associated with a significant reduction in allcause mortality (39). Luc et al reported that compared to patients who had PCI, those who underwent CABG had a higher early mortality and overall mortality, but there was no difference in cardiac-related mortality in heart transplant recipients with coronary allograft vasculopathy (40).

The 5-year survival rate in revascularization interventions is greater than medical therapy. Also, this rate is different between revascularization interventions and is more in PCI intervention than CABG. A previous network meta-analysis comparing percutaneous coronary intervention with medical treatment was limited to the inclusion of early generation paclitaxel eluting stents and sirolimus eluting stents and observed no benefit in medical treatment in terms of survival (41). In another study, long-term survival was not significantly different between PCI and
CABG among patients in whom coronary revascularization was clinically indicated, and both procedures were technically feasible for these patients (42). Thus, despite this similarity in long-term survival, several distinct differences between these alternative coronary revascularization procedures may affect the choice of procedure. The most important finding of this analysis was that several revascularization techniques, such as coronary artery bypass grafting and percutaneous coronary intervention, were found to be associated with improved survival compared to an initial strategy of medical therapy alone among patients with ischemic cardiomyopathy.

PCI and CABG had improved QoL. Both were significantly better than medical therapy but did not differ from each other. These findings were consistent with those of Blankenship et al (43) who concluded that PCI decreases angina and the need for anti-anginal medications and increases exercise capacity and QoL, compared to baseline status and medical therapy without PCI(Jokinen et al) (44). However, these authors examined only PCI and only $\mathrm{CABG}$, respectively. A discussion exists in the literature regarding the instruments used in QoL research. In this study, all studies used valid and reliable measures. However, most of them used generic rather than disease specific measures. As there has been no agreed upon definition of QoL, results produced by various measurements may vary significantly because each instrument may include mutual as well as different domains, depending on the 
theoretical framework used by the authors.

Results of this study indicated that PCI and CABG, as initial treatments, were associated with higher utility and patient satisfaction compared to MT. Also, between the revascularization interventions, results of the 2 included studies were different and contradictory with regards to patient satisfaction. The overall results of this study are not directly comparable with those of previous studies because we applied this research tool in a study that compared 3 therapeutic strategies simultaneously. Hlatky MA et al reported that utility was more favorable among CABG patients for the first year, which was different from our results, although the values became similar thereafter (45).

\section{Conclusion}

This study showed that revascularization interventions in all indices are more effective than medical therapy. Also, between revascularization interventions, PCI in cardiovascular mortality and 5-year survival were more effective than CABG. Also, in terms of quality of life, CABG was more effective than PCI. However, with regards to patient satisfaction index, the results of the 2 included studies were different contradictory. Thus, both CABG and PCI are reasonable options for patients with ischemic cardiomyopathy. Current evidence dictates that despite advances in $\mathrm{CABG}$, patients with ischemic cardiomyopathy are better served with PCI intervention.

\section{Limitations}

As with any meta-analysis, the conclusions drawn from such data are subject to the limitations of the original studies. Similarly, due to the lack of patient level data, we could not account for different follow-up times and for censoring or drop-outs by performing any meaningful survival analysis.

\section{Acknowledgments}

This article was part of a Ph.D. thesis in Health Economics at Iran University of Medical Sciences (IUMS), (grant number: IUMS/SHMIS 9321504003). The authors would like to thank Iran University of Medical Sciences for providing financial support for this study.

\section{Conflict of interests}

The authors declare that they have no competing interests.

\section{References}

1.Wijeysundera HC, Bennell MC, Qiu F, Ko DT, Tu JV, Wijeysundera DN, et al. Comparative-effectiveness of revascularization versus routine medical therapy for stable ischemic heart disease: a populationbased study. J Gen Intern Med. 2014;29(7):1031-9.

2.Marui A. Coronary-artery bypass surgery plus medical therapy is associated with longer survival over 10 years than medical therapy alone in patients with ischaemic cardiomyopathy. Evid Based Med. 2017;22(1):32.

3.Nudi F, Procaccini E, Versaci F, Giordano A, Pinto A, Neri G, et al. Impact of coronary revascularization on the clinical and scintigraphic outlook of patients with myocardial ischemia. $\mathrm{j}$ cardivasc med. 2017;18(6):404-9.
4.Shah BR, Velazquez E, Shaw LK, Bart B, O'Connor C, Wagner GS. Revascularization improves survival in ischemic cardiomyopathy regardless of electrocardiographic criteria for prior small-to-medium myocardial infarcts. Am Heart J. 2002;143(1):111-7.

5.Velazquez EJ, Williams JB, Yow E, Shaw LK, Lee KL, Phillips HR, et al. Long-term survival of patients with ischemic cardiomyopathy treated by coronary artery bypass grafting versus medical therapy. Ann Thorac Surg. 2012;93(2):523-30.

6.Bertomeu-Gonzalez V, Bouzas-Mosquera A, Kaski JC. Role of trimetazidine in management of ischemic cardiomyopathy. Am J Cardiol. 2006;98(5):19-24.

7.Lin T, Hasaniya NW, Krider S, Razzouk A, Wang N, Chiong JR. Mortality Reduction With $\beta$-Blockers in Ischemic Cardiomyopathy Patients Undergoing Coronary Artery Bypass Grafting. Congest Heart Fail. 2010;16(4):170-4.

8.Boden WE, O'Rourke RA, Teo KK, Hartigan PM, Maron DJ, Kostuk WJ, et al. Optimal medical therapy with or without PCI for stable coronary disease. N Engl J Med. 2007;356(15):1503-16.

9. Stanley WC, Recchia FA, Lopaschuk GD. Myocardial substrate metabolism in the normal and failing heart. Physiol Rev. 2005;85(3):1093-129.

10.Rezapour A, Faradonbeh SB, Alipour V, Yusefvand M. Effectiveness of revascularization interventions compared with medical therapy in patients with ischemic cardiomyopathy: A systematic review protocol. Medicine (Baltimore). 2018;97(10).

11.Carson P, Wertheimer J, Miller A, O'Connor CM, Pina IL, Selzman $\mathrm{C}$, et al. The STICH trial (Surgical Treatment for Ischemic Heart Failure): mode-of-death results. JACC: Heart Failure. 2013;1(5):400-8.

12.Khera S, Panza JA. Surgical revascularization in older adults with ischemic cardiomyopathy. heart fail clin. 2017;13(3):571-80.

13. Meads C, Cummins C, Jolly K, Stevens A, Burls A, Hyde C. Coronary artery stents in the treatment of ischaemic heart disease: a rapid and systematic review. Health Technol Assess. 2000;4(23):1153.

14.Graham MM, Ghali WA, Faris PD, Galbraith PD, Norris CM, Knudtson ML. Survival after coronary revascularization in the elderly. Circulation. 2002;105(20):2378-84.

15.O'Connor CM, Velazquez EJ, Gardner LH, Smith PK, Newman MF, Landolfo KP, et al. Comparison of coronary artery bypass grafting versus medical therapy on long-term outcome in patients with ischemic cardiomyopathy (a 25-year experience from the Duke Cardiovascular Disease Databank). Am J Cardiol. 2002;90(2):101-7.

16. Cleland JGF, Walker A. Is medical treatment for angina the most cost-effective option? Eur Heart J. 1997;18(suppl_B):35-42.

17.Hueb W, Lopes N, Gersh BJ, Soares PR, Ribeiro EE, Pereira AC, et al. Ten-year follow-up survival of the Medicine, Angioplasty, or Surgery Study (MASS II): a randomized controlled clinical trial of 3 therapeutic strategies for multivessel coronary artery disease. Circulation. 2010;122(10):949-57.

18.Claude J, Schindler C, Kuster GM, Schwenkglenks M, Szucs T, Buser $\mathrm{P}$, et al. Cost-effectiveness of invasive versus medical management of elderly patients with chronic symptomatic coronary artery disease: findings of the randomized trial of invasive versus medical therapy in elderly patients with chronic angina (TIME). Eur Heart J. 2004;25(24):2195-203.

19.Weiss S, Weintraub W. Revascularization vs. medical therapy in stable ischemic heart disease. Progress Cardiovasc Dis. 2015;58(3):299-305.

20.Edlin R, McCabe C, Hulme C, Hall P, Wright J. Cost effectiveness modelling for health technology assessment: Springer; 2015.

21.Raja V, Wiegn P, Obel O, Christakopoulos G, Christopoulos G, Rangan BV, et al. Impact of chronic total occlusions and coronary revascularization on all-cause mortality and the incidence of ventricular arrhythmias in patients with ischemic cardiomyopathy. Am J Cardiol. 2015;116(9):1358-62.

22.Hutton B, Salanti G, Caldwell DM, Chaimani A, Schmid CH, Cameron C, et al. The PRISMA extension statement for reporting of systematic reviews incorporating network meta-analyses of health care interventions: checklist and explanations. Ann Intern Med. 2015;162(11):777-84

23. Shah BR, Velazquez E, Shaw LK, Bart B, O'Connor C, Wagner GS. Revascularization improves survival in ischemic cardiomyopathy regardless of electrocardiographic criteria for prior small-to-medium myocardial infarcts. Am Heart J. 2002;143(1):111-7.

24.Kwon DH, Hachamovitch R, Popovic ZB, Starling RC, Desai MY, 
Flamm SD, et al. Survival in patients with severe ischemic cardiomyopathy undergoing revascularization versus medical therapy: association with end-systolic volume and viability. Circulation. 2012;126(11 suppl 1):S3-S8.

25.Mark DB, Knight JD, Velazquez EJ, Wasilewski J, Howlett JG, Smith PK, et al. Quality-of-Life Outcomes in Surgical Treatment of Ischemic Heart Failure Quality-of-Life Outcomes With Coronary Artery Bypass Graft Surgery in Ischemic Left Ventricular Dysfunction: A Randomized Trial. Ann Intern Med. 2014;161(6):392.

26.Sabaghian H, Holakoui K. Epidemiology. Tehran: Gap; 2013.

27.Graham MM, Norris CM, Galbraith PD, Knudtson ML, Ghali WA. Quality of life after coronary revascularization in the elderly. Eur Heart J. 2006;27(14):1690-8.

28.Jung RE, Pjetursson BE, Glauser R, Zembic A, Zwahlen M, Lang NP. A systematic review of the 5-year survival and complication rates of implant-supported single crowns. Clin Oral Implants Res. 2008;19(2):119-30.

29.Rockwood TH, Church JM, Fleshman JW, Kane RL, Mavrantonis C, Thorson AG, et al. Fecal incontinence quality of life scale. Dis Colon Rectum. 2000;43(1):9-16.

30.Williams LS, Weinberger M, Harris LE, Clark DO, Biller J. Development of a stroke-specific quality of life scale. Stroke. 1999;30(7):1362-9.

31.Rezazadeh E, Hachesu PR, Rezapoor A, Alireza K. Evidence-based medicine: going beyond improving care provider viewpoints, using and challenges upcoming. J Evid Based Med. 2014;7(1):26-31.

32.Brandão SMG, Hueb W, Ju YT, de Lima ACP, Polanczyk CA, Cruz $\mathrm{LN}$, et al. Utility and quality-adjusted life-years in coronary artery disease: Five-year follow-up of the MASS II trial. Medicine. 2017;96(50).

33.Prakash B. Patient satisfaction. J Cutan Aesthet Surg. 2010;3(3):151.

34.Jadad AR, Moore RA, Carroll D, Jenkinson C, Reynolds DJM, Gavaghan DJ, et al. Assessing the quality of reports of randomized clinical trials: is blinding necessary? Control Clin Trials. 1996;17(1):112.

35. Shabaninejad H, Asgharzadeh A, Rezaei N, Rezapoor A. A comparative study of intravenous immunoglobulin and subcutaneous immunoglobulin in adult patients with primary immunodeficiency diseases: a systematic review and meta-analysis. Expert Rev Clin Immunol. 2016;12(5):595-602.

36.Huedo-Medina TB, Sánchez-Meca J, Marín-Martínez F, Botella J. Assessing heterogeneity in meta-analysis: Q statistic or $\mathrm{I}^{2}$ index? Psychol Methods. 2006;11(2):193.

37.Drummond MF, Sculpher MJ, Claxton K, Stoddart GL, Torrance GW. Methods for the economic evaluation of health care programmes: Oxford University Press; 2015.

38.Pursnani S, Korley F, Gopaul R, Kanade P, Chandra N, Shaw RE, et al. Percutaneous coronary intervention versus optimal medical therapy in stable coronary artery disease: a systematic review and metaanalysis of randomized clinical trials. Circ-Cardiovasc Inte. 2012;5(4):476-90.

39.Schömig A, Mehilli J, de Waha A, Seyfarth M, Pache J, Kastrati A. A meta-analysis of 17 randomized trials of a percutaneous coronary intervention-based strategy in patients with stable coronary artery disease. J Am Coll Cardiol. 2008;52(11):894-904.

40.Luc JGY, Choi JH, Rizvi S-SA, Phan K, Escrivà EM, Patel S, et al. Percutaneous coronary intervention versus coronary artery bypass grafting in heart transplant recipients with coronary allograft vasculopathy: a systematic review and meta-analysis of 1,520 patients. Ann Thorac Cardiovasc Surg. 2018;7(1):19.

41.Trikalinos TA, Alsheikh-Ali AA, Tatsioni A, Nallamothu BK, Kent DM. Percutaneous coronary interventions for non-acute coronary artery disease: a quantitative 20-year synopsis and a network metaanalysis. Lancet. 2009;373(9667):911-8.

42.Bravata DM, Gienger AL, McDonald KM, Sundaram V, Perez MV, Varghese R, et al. Systematic review: the comparative effectiveness of percutaneous coronary interventions and coronary artery bypass graft surgery. Ann Intern Med. 2007;147(10):703-16.

43.Blankenship JC, Marshall JJ, Pinto DS, Lange RA, Bates ER, Holper $\mathrm{EM}$, et al. Effect of percutaneous coronary intervention on quality of life: a consensus statement from the Society for Cardiovascular Angiography and Interventions. Catheter Cardiovasc Interv. 2013;81(2):243-59.

44.Jokinen JJ, Hippeläinen MJ, Turpeinen AK, Pitkänen O, Hartikainen JEK. Health-Related Quality of Life After Coronary Artery Bypass
Grafting: A Review of Randomized Controlled Trials. J Card Surg. 2010;25(3):309-17.

45.Hlatky MA, Boothroyd DB, Melsop KA, Brooks MM, Mark DB, Pitt $\mathrm{B}$, et al. Medical costs and quality of life 10 to 12 years after randomization to angioplasty or bypass surgery for multivessel coronary artery disease. Circulation. 2004;110(14):1960-6. 